\title{
La dépression - un système dynamique complexe
}

\section{Günter Schiepek}

Psychotherapie-Wissenschaft 10 (2) 592020

www.psychotherapie-wissenschaft.info

CC BY-NC-ND

https://doi.org/10.30820/1664-9583-2020-2-59

Mots clés : psychothérapie personnalisée, suivi de processus, schémas thérapeutiques, transitions d'ordre, indicateurs d'alerte précoce, schémas de processus neuronaux, neurostimulation non invasive

Le présent article traite des résultats récents de la recherche sur la dépression, notamment en ce qui concerne le traitement psychothérapeutique de la dépression. À la demande des rédacteurs, il s'agissait également de fournir une synthèse des développements scientifiques et pratiques qui ont lieu à l'Institut de Recherche sur la Synergetique et la Psychothérapie de l'Université Médicale Privée Paracelsus de Salzbourg et dans les groupes de recherche qui coopèrent avec cet institut. L'un des axes de ces développements réside dans la personnalisation de la psychothérapie et concerne à la fois les nouvelles méthodes de conception des cas (par exemple, la modélisation du système idiographique et sa visualisation par ordinateur) et les méthodes de contrôle et de rétroaction à haute fréquence des processus. La modélisation du système idiographique est une méthode avec laquelle le thérapeute et le patient développent un modèle de réseau graphique des cognitions, des émotions, du comportement et des conditions environnementales pertinentes pour le problème individuel dans un processus de coopération. Le système de navigation synergétique (SNS) basé sur Internet et sur des applications a été développé et testé pour l'enregistrement et l'analyse des processus thérapeutiques.

Dans une étude portant sur 328 et patientes souffrant de troubles affectifs (principalement différents diagnostics de dépression), le déroulement de la psychothérapie a été enregistré avec des mesures quotidiennes du processus. Les progressions montrent des sauts discontinus entre des modèles dynamiques (appelés transitions d'ordre), qui sont initiés par une instabilité critique accrue (complexité dynamique) et une synchronisation temporaire des éléments de la feuille de processus utilisée. Une instabilité critique accrue est également prédictive d'un meilleur succès thérapeutique. Dans le groupe des répondants, l'intensité de l'instabilité critique est en corrélation avec le résultat (réduction des symptômes), mais pas chez les non-répondants. Une étude sur des patients et patientes dépressifs comorbides souffrant de troubles obsessionnels compulsifs a montré qu'en plus des améliorations cliniques, la dynamique de la connectivité fonctionnelle des réseaux neuronaux change également. Les examens répétés d'IRMf, des états de repos montrent que la flexibilité et la fluidité de la neurodynamique sont associées négativement à la psychopathologie (par exemple, l'intensité de la dépression enregistrée avec le BDI-II), mais augmentent au cours de la psychothérapie. La rigidité neuronale et cognitivo-affective est un marqueur important de la psychopathologie, mais s'avère modifiable sur le plan thérapeutique. Enfin, une évaluation est faite des possibilités de combiner la neurostimulation non invasive avec la psychothérapie. Le défi consiste ici à influencer la dynamique très souple des structures de connectivité neurale dans le sens d'un système de rétroaction (à la demande).

\section{L'auteur}

Günter Schiepek, professeur à l'université, Dr. phil. Dr. phil. habil, est directeur de l'Institut de Recherche sur la Synergetique et la Psychothérapie de l'Université Privée de Médecine Paracelse (Paracelsus Medizinischen Privatuniversität / PMU) de Salzbourg, professeur à la PMU et à l'Université Ludwig-Maximilian de Munich, professeur invité à l'Université Sapienza de Rome, Directeur général du Centre pour les systèmes complexes (Stuttgart/Salzbourg), membre de l'Académie Européenne des Sciences et des Arts (Academia Scientiarium et Artium Europaea) et membre honoraire de la Société systémique (organisation faîtière allemande pour la recherche, la thérapie, la supervision et le conseil systémiques).

\section{Contact}

Prof. Dr. Günter Schiepek

Institut für Synergetik und Psychotherapieforschung Universitätsklinik für Psychiatrie, Psychotherapie und Psychosomatik

Paracelsus Medizinische Privatuniversität

Ignaz Harrer Str. 79

A-5020 Salzburg

E-Mail : guenter.schiepek@ccsys.de 